\title{
The Ubiquitous DBMS
}

\author{
Kyu-Young Whang \\ Department of Computer Science, KAIST \\ kywhang@mozart.kaist.ac.kr
}

\begin{abstract}
Recent widespread use of mobile technologies and advancement in computing power prompted strong needs of database systems that can be used in small devices such as sensors, cellular phones, PDA, ultra PCs, and navigators. We call database systems that are customizable from small-scale applications for small devices to large-scale applications such as large-scale search engines ubiquitous database management systems (UDBMSs). In this talk, we first review requirements of UDBMSs. The requirements we identified include selective convergence (or "devicetization"), flash-optimized storage systems, data synchronization, supportability of unstructured/semi-structured data, and complex database operations. We then review existing systems and research prototypes. We first review the functionality of UDBMSs including the footprint size, support of standard SQL, supported data types, transactions, concurrency control, indexing, and recovery. We then review the supportability of requirements by those UDBMSs surveyed. We highlight ubiquitous features of a family of Odysseus systems that have been under development at KAIST for over 20 years. Functionalities of Odysseus can be "devicetized" or customized depending on the device types and applications as in Odysseus/Mobile for small devices, Odysseus/XML for unstructured/semistructured data, Odysseus/GIS for map data, and Odysseus/IR for large-scale search engines. We finally present research topics that are related to the UDBMSs.
\end{abstract}

